\title{
Caracterización morfológica in situ de Ojushte (Brosimum alicastrum Swartz) y su incidencia en la selección de germoplasma de alto potencial nutricional en El Salvador
}

\author{
Molina-Escalante, $\mathrm{MO}^{1}$; Castillo-Guerra, $\mathrm{LO}^{1}$; Parada-Berrios, $\mathrm{FA}^{2}$; \\ Lara-Ascencio, $F^{3}$; Linares, $A Y^{4}$
}

1. Tesista de la Facultad de Ciencias Agronómicas, UES.

2. Docente asesor del departamento de Ritotecnica, Facultad de Ciencias Agronómicas, UES; e-mail: faparadaberrios@yahoo.com

3. Docente asesor del departamento de Protección Vegetal, Facultad de Ciencias Agronómicas, UES; e-mail: fcolaraa@yahoo.es

4.Docente asesor del departamento de Química Agrícola, Facultad de Ciencias Agronómicas, UES; e-mail: yani_linares@hotmail.com

\section{Resumen}

Recepción: 2014-04-23

Aceptación: 2014-05-23

Con el objetivo de identificar y caracterizar morfológicamente in situ árboles de Ojushte, para seleccionar germoplasma de alto potencial nutricional, se ejecutó la investigación de febrero 2013 a febrero de 2014. Esta labor incluyó giras a lugares con poblaciones naturales de la especie, distribuidas en diversas localidades de El Salvador: San Pedro, Chirilagua (San Miguel); Área Natural Protegida Nancuchiname, San Marcos Lempa (Usulután); La Bermuda, Suchitoto (Cuscatlán); San Laureano, Ciudad Delgado y Universidad de El Salvador (San Salvador); San Isidro y Área Natural Protegida Plan de Amayo, Caluco (Sonsonate) y Upatoro (Chalatenango). En la caracterización de los árboles se tomaron en cuenta caracteres cualitativos y cuantitativos. Para la interpretación de los datos se utilizó estadística descriptiva y análisis multivariado, en donde se utilizó el programa SPSS versión 20.

Se caracterizaron un total de 30 árboles productivos de Ojushte, de los cuales 23 resultaron con atributos sobresalientes en cuanto a características químicas de semilla, proteína, con rango de 12.25 - 13.99\%; hierro con 31 74.09 mg.l-1; y zinc con 11.6 - 18.34 mg.l-1 (los rangos fueron calculados por medio de probabilidad estadística), los cuales pueden ser utilizados en programas de producción, alimentación humana y mejoramiento genético. Por otra parte la variabilidad genética cuantitativa de los árboles estudiados estuvo determinada por el $42 \%$ de los descriptores, mostrando coeficientes de variación mayores al 23\%. El método multivariado permitió agrupar en seis componentes a 23 árboles de Ojushte, en donde cada grupo se caracterizó por mostrar perfiles muy afines entre sí y diferentes entre los grupos. Del total de árboles estudiados se tomó una muestra para realizar el análisis bromatológico correspondiente.

Palabras clave: Ojushte, Brosimum alicastrum, seguridad alimentaria, nutrición humana

\begin{abstract}
With the objective to identify and charaterize morphologically in situ Ojushte trees, to select germplasm with high nutritional potential, it was execute this research from February 2013 to February 2014. This task included trips to places where there are natural populations of this specie in El Salvador: San Pedro, Chirilagua (San Miguel), Nancuchiname Natural Protected Area, San Marcos Lempa (Usulután; La Bermuda, Suchitoto (Cuscatlán); San Laureano, Ciudad Delgado and Universidad de El Salvador (San Salvador); San Isidro and Plan de Amayo Natural Protected Area, Caluco (Sonsonate) and Upatoro (Chalatenango). For the characterization of the trees, qualitative and quantitative characters were taken For the interpretation of the data, the descriptive statistics method and the multivariated analysis were used. For the last one, it was used the software SPSS version 2.0.

A total of 30 Ojushte trees was characterized, when 23 of them resulted with protruding attributes in the chemical characteristics of the seed and protein with a range of $12.25-13.99 \%$, iron with $31-74.09$ mg.l-1, and zinc with 11.6 - $18.34 \mathrm{mg} .1-1$ (the ranges were calculated through statistics probability), that can be used in production programs, human feeding and genetic improvement. On the other hand, the quantitative genetic variability of the studied trees was determined by the $42 \%$ of the descriptors showing coefficients of variation higher than $23 \%$. The multivariated method allowed to group six components to 23 Ojushte trees where each group had the characteristic of showing very similar profiles among them and different from the groups. A sample was taken from them in order to carry out the corresponding nutritional analysis.
\end{abstract}

Keywords: Ojushte, Brosimum alicastrum, food security, human nutrition 


\section{Introducción}

El Ojushte (Brosimum alicastrum Swartz) pertenece a la familia Moraceae, con distribución desde México hasta Costa Rica, siendo propio de alturas que oscilan de 0 - 1000 metros sobre el nivel del mar (BOSTIC, citado por Linares e Ibáñez, 1992). Este es un árbol mediano a grande, siempre verde, que alcanza una altura de $34 \mathrm{~m}$ o más; y un diámetro a la altura del pecho de un metro. Los árboles grandes muestran contrafuertes, la corteza es algo lisa, de color gris oscuro, con grietas finas verticales (Witsberguer et al. 1982).

Los frutos del Ojushte son bayas de 2 a $3 \mathrm{~cm}$ de diámetro, globosas con pericarpio carnoso. Presenta una coloración verde cuando está inmaduro, y de verde amarillento a anaranjado rojizo al madurarse. Tiene un sabor y olor dulces (Morales y Herrera 2009, Mendoza y Santillana 2012). Cada fruto contiene una semilla de 0.90 a $1.30 \mathrm{~cm}$ de diámetro polar por 1.60 a $2 \mathrm{~cm}$ de diámetro ecuatorial. Son de forma esférica o aplanada, cubiertas de una testa papirácea de color café - blanquecino, que al secarse se desprende sola, dejando una semilla de dos cotiledones asimétricos de coloración verdusca. La consistencia del endospermo crudo es semejante al de una zanahoria (Aragón 1990, Mendoza y Santillana 2012, Morales y Herrera, 2009).

En el estado de Yucatán, México, se ha observado que esta especie florece de no- viembre a febrero. En la región sur lo hacen desde el mes de noviembre; mientras que en la región centro y norte su floración inicia en los meses de enero y febrero (Morales y Herrera, 2009). Su fructificación varía de febrero a octubre en América Central. En ciertas áreas puede haber dos picos de producción (ejemplo, en Honduras de febrero a abril y de agosto a octubre) (CATIE, 2003).

La Academia de Ciencias de Estados Unidos (1975) incluyó al Ojushte en la lista de plantas tropicales subexplotadas con promisorio valor económico, pues a cada una de sus partes se le puede dar un uso práctico. La semilla tostada y molida se utiliza como un sustituto del café sin cafeína; hervida y molida, se usa como masa muy nutritiva para hacer tortillas. Al látex y corteza se le atribuyen propiedades medicinales, mientras que su madera es utilizada para múltiples fines como la elaboración de muebles y artesanías (Meiners et al, 2009).

\section{Materiales y métodos}

\section{a. Localización}

La caracterización morfológica de Ojushte (Brosimum alicastrum Swartz) se realizó en el período comprendido entre febrero de 2013 y febrero de 2014, realizando expediciones de colecta en Áreas Naturales Protegidas (ANP): Caluco, Sonsonate y Nancuchiname, Usulután. Asimismo se visitaron otros lugares de prevalencia natural de la especie en los depar- 
tamentos de San Salvador, San Miguel, Cuscatlán y Chalatenango. Los pisos altitudinales de los árboles caracterizados fluctuaron entre los $0-800 \mathrm{msnm}^{5}$ (Figura 1).

\section{1 Material experimental}

En total se identificaron 30 árboles productivos de Ojushte, los cuales producen en la época seca y época lluviosa.
La caracterización de frutos solamente se realizó a 14 árboles; mientras que la individualización de semilla y análisis bromatológico se practicó a 23 de ellos. Por otra parte, a siete árboles no se les realizó identificación de fruto ni semilla debido a que cuando se visitó el lugar no era época de cosecha.

\section{Cuadro resumen de localización y altura de árboles de Ojushte}

\begin{tabular}{|l|c|c|}
\hline Lugar de identificación de Ojushte & $\begin{array}{c}\text { Altura } \\
\text { msnm }\end{array}$ & Número de árboles muestreados \\
\hline $\begin{array}{l}\text { Área Natural Protegida Plan de Amayo - } \\
\text { Caluco, Sonsonate }\end{array}$ & 344.25 & 8 \\
\hline San Isidro, Sonsonate & 545 & 3 \\
\hline San Pedro - Chirilagua, San Miguel & 145.33 & 3 \\
\hline La Bermuda - Suchitoto, Cuscatlán & 543.5 & 4 \\
\hline $\begin{array}{l}\text { Área Natural Protegida de Nancuchi- } \\
\text { name, San Marcos Lempa }\end{array}$ & 6 & 3 \\
\hline $\begin{array}{l}\text { San Laureano - Ciudad Delgado, San } \\
\text { Salvador }\end{array}$ & 598.5 & 1 \\
\hline Villa Belén - Apopa, San Salvador & 472 & 1 \\
\hline Universidad de El Salvador, San Salvador & 750 & 4 \\
\hline Upatoro, Chalatenango & 517 & 3 \\
\hline
\end{tabular}

Figura 1. Lugares y datos altitudinales de árboles estudiados. Materiales y métodos (2013) 


\section{b. Evaluación del germoplasma de Ojushte}

A fin de realizar la identificación del Brosimum alicastrum Swartz, se elaboró un descriptor especifico, tomando como base el utilizado por Torres Calderón (2007) para el mamey, el descriptor de aguacate, empleado por Rodríguez y Gutiérrez (2012), y la clave, propuesta por la FAO, usada por Aragón (1990), para el descriptor de Ojushte. También, se asignó un código para cada árbol, el cual se estructuró utilizando el nombre del lugar de recolección (finca, comunidad, parque o Área Natural Protegida) y el número con base a un orden correlativo.

Cada árbol, del cual se obtuvo muestras, fue georeferenciado con un sistema de posicionamiento global (GPS) de navegación marca GARMIN, modelo GPSMAP 60 CSx, con precisión de $6 \mathrm{~m}$, configurado con los datos Datum WGS 84 y sistema de coordenadas geográficas.

\section{c. Variables en estudio}

Las variables cualitativas fueron: hábito de crecimiento, forma de gamba, color de tallo, color de fruto, época de floración y cosecha; duración de cosecha, forma de fruto y semilla: forma de hoja y pubescencia. Por otra parte, las variables cuantitativas fueron: edad del árbol, diámetro del tronco a la altura del pecho (DAP), diámetro de copa, altura del árbol, longitud y ancho de lámina foliar; área foliar, diámetro polar y ecuatorial de fruto $\mathrm{y}$ semilla, peso de fruto y semilla, y análisis bromatológico de la misma.

\section{d. Análisis bromatológico}

El análisis bromatológico se realizó en los Laboratorios de Química Agrícola de la Facultad de Ciencias Agronómicas de la Universidad de El Salvador. Se utilizaron 100g de semilla por cada árbol estudiado. A cada muestra se le determinó humedad parcial, humedad total, proteína, fibra, grasa, hierro y zinc.

\section{e. Metodología estadística}

Para la interpretación de variables cualitativas se hizo uso de estadística descriptiva, por medio de frecuencia absoluta y frecuencia relativa. Mientras que para las variables cuantitativas se aplicó estadística simple (media, desviación estándar y coeficiente de variación), y análisis multivariado, específicamente análisis de correlación, componentes principales y conglomerados. Todo ello se realizó utilizando el programa SPSS versión 20, que es un sistema global para el análisis de datos.

\section{Resultados y discusión}

\section{a. Forma de gamba, color de tallo y color de fruto.}

Según el descriptor utilizado se encontraron cinco formas de gambas, en donde el $64 \%$ de los árboles encontrados presentaron una for- 
ma IV. Esta se caracterizó por mostrar las dimensiones siguientes: altura mayor a $1.50 \mathrm{~m}$, largo horizontal de $0.50 \mathrm{~cm}$ y forma angular; mientras que un 3\% de los árboles corresponden a las forma I y $\mathrm{V}$.

Esta última forma no es reportada por la clave para el descriptor de Ojushte propuesto por la FAO - usada por Aragón (1990) -; pero se incluye como producto de esta investigación, por haberse encontrado en el árbol Upatoro 4. Este espécimen presentó gambas de $0.75 \mathrm{~cm}$ de altura, largo horizontal de $0.40 \mathrm{~cm}$ y forma achatada.

Con respecto al color de tallo, se encontró que el $76.66 \%$ de los árboles presentaron color café oscuro - grisáceo y el 23.34\% color café claro - grisáceo. Witsberguer et al (1982) hace mención de una sola coloración de la especie, la cual es café oscuro - grisáceo; mientras que Morales y Herrera (2009) hacen mención de una coloración diferente, que es gris clara a parda. Entre los árboles caracterizados se encontró que estos tallos color café claro - grisáceo fructifican (floración - cosecha) en la época seca e inicios de la época lluviosa, como el caso de los árboles San Isidro 2 y San Isidro 3.

En cuanto al color del fruto, se determinó que en el país existen tres colores diferentes, los cuales son: amarillo, rojo y verde. El 73.40\% de los árboles presentaron fruto color verde, $13.30 \%$ frutos rojo, y el $13.30 \%$ frutos amari- 1lo. Los árboles con frutos amarillos y rojos, solo se encontraron en la época seca e inicio de la época lluviosa; mientras que los frutos verdes pertenecen a la época lluviosa. Al respecto Mendoza y Santillana (2012) mencionan que el fruto presenta una coloración verde amarillento $\mathrm{u}$ anaranjado, o rojo en completa madurez, cubierta en la superficie de numerosas escamas blancas.

\section{b. Época de floración y cosecha}

El periodo de floración del Brosimum alicastrum Swartz, ocurre entre los meses de noviembre a junio, y el periodo de producción de fruto se da de enero a agosto. Esto tiene concordancia con el desarrollo del fruto (desde flor hasta cosecha), que según lo determinado es de 60 a 75 días (determinado a través del monitoreo del árbol UES 1). Puleston, citado por Aragón (1990), hace mención de un periodo similar de fructificación del Brosimum que es de 50 a 75 días.

Se determinó que el 73.33\% de los árboles encontrados producen fruto en la época lluviosa (63.33\% invierno temprano (mayo - junio) y $10 \%$ invierno intermedio (julio - agosto)); mientras que un $26.6 \%$ de ellos producen en la época seca (10\% verano intermedio (enero - febrero) y $16.6 \%$ verano tardío (marzo abril)). Asimismo, se determinó que los meses de mayor abundancia para las dos épocas son febrero - marzo (árboles de época seca) y junio - julio (para árboles de época lluviosa). 
Al respecto, Morales y Herrera (2009) mencionan que el período de mayor abundancia de Ojushte es en los meses de enero - junio. Morales y Herrera (2009) mencionan un intervalo de cosecha similar, el cual varía únicamente por un mes; mientras que CATIE (2003) indica que la fructificación ocurre de febrero a octubre en América Central. Este dato no coincide con los hallazgos obtenidos en El Salvador, ya que hace mención de dos meses (septiembre - octubre), en los cuales no se encontró árboles en producción. Eso hace pensar que, probablemente hace referencia a otros lugares de la región.

\section{c. Forma de la hoja y pubescencia en el envés}

En cuanto a la forma de la hoja, se encontró que 18 árboles presentaron hojas con forma oblonga - lanceolada y 12 en forma elíptica. Estos resultados coinciden con los mencionados por Peters, citado por Mendoza y Santillana (2012), quien hace mención de las formas oblonga - lanceolada a ovadas o elípticas.

Los árboles que presentaron un envés glabro fueron: Plan de Amayo 1, Plan de Amayo 2, Zunsal 1, Zunsal 2, Isidro 1, Isidro 2 e Isidro 3. Mientras que el resto presentó un envés escasamente pubescente.

Una observación realizada durante la investigación fue que la mayoría de estos árboles fructificaron en la época seca o inicio de la época lluviosa, a excepción del árbol Plan de
Amayo 3, que fructificó en la misma época del árbol Zunsal 2, pero que presentó un envés escasamente pubescente. Ante esto, Witsberguer et al. (1982) menciona que el envés de la hoja de Ojushte puede ser glabro o escasamente pubescente.

\section{d. Diámetro polar y ecuatorial de fruto y semilla}

En la figura 2 se observa que el árbol Plan de Amayo 4 presentó los mayores valores de diámetro ecuatorial y polar de fruto con $2.31 \mathrm{y}$ $2.34 \mathrm{~cm}$, respectivamente. Por otra parte, el menor diámetro lo presentó el árbol Plan de Amayo 3 , con valores que van de 1.43 a $1.23 \mathrm{~cm}$.

El diámetro ecuatorial promedio de los 14 árboles fue de $2.06 \mathrm{~cm}$, con una desviación estándar de $0.22 \mathrm{~cm}$ y un coeficiente de variación de 10.67\%. Asimismo, el 85\% de los árboles se encontró dentro del rango 1.84 $2.28 \mathrm{~cm}$; mientras que el diámetro polar promedio fue de $1.97 \mathrm{~cm}$, con una desviación estándar de $0.28 \mathrm{~cm}$ y un coeficiente de variación de $14.21 \%$.

A nivel general, el 79\% de los árboles se encontraron dentro del rango $1.69-2.25 \mathrm{~cm}$. Asimismo, Aragón (1990) en la caracterización preliminar de Ojushte, realizada en el bosque húmedo sub - tropical cálido de Peten, Guatemala, determinó que el diámetro promedio del fruto fue de $2.27 \mathrm{~cm}$, con un mínimo de $1.9 \mathrm{~cm}$ y máximo $3.0 \mathrm{~cm}$, con una 
desviación estándar de $0.20 \mathrm{~cm}$. Estos datos sin especificar si dichos valores son referidos a diámetros polares o ecuatoriales.

Con respecto al diámetro polar de semilla el árbol Zunsal 1, esta presentó el mayor valor con $1.40 \mathrm{~cm}$, y el árbol San Isidro 1 presentó el menor diámetro con $0.94 \mathrm{~cm}$ (Figura 3). El diámetro polar promedio fue de $1.16 \mathrm{~cm}$, desviación estándar de $0.14 \mathrm{~cm}$ y coeficiente de variación de $12 \%$. Por otra parte, el $61 \%$ de los árboles se encontró dentro del rango 1.02 - $1.3 \mathrm{~cm}$. Al respecto Mendoza y Santillana (2012) mencionan valores muy afines, que es de $0.9-1.3 \mathrm{~cm}$ de diámetro polar.

En cuanto al diámetro ecuatorial de semilla, el árbol Zunsal 1 presentó el rango mayor con $1.77 \mathrm{~cm}$, seguido del San Laureano 1 con un valor de $1.7 \mathrm{~cm}$. Por su parte, el árbol Plan de
Amayo 3 presentó el menor diámetro con $1.24 \mathrm{~cm}$ (Figura 3).

El diámetro ecuatorial promedio de los 23 árboles fue de $1.50 \mathrm{~cm}$, con una desviación estándar de $0.15 \mathrm{~cm}$ y un coeficiente de variación de $10 \%$. A nivel general, el $65 \%$ de los árboles se encontraron dentro del rango de $1.35-1.6 \mathrm{~cm}$. Al respecto Mendoza y Santillana (2012) mencionan valores afines a los encontrados, los cuales son de 1.6 a $2.0 \mathrm{~cm}$ de diámetro ecuatorial.

\section{Gráfica sobre diámetro polar y ecuatorial de fruto de los árboles de Ojushte}

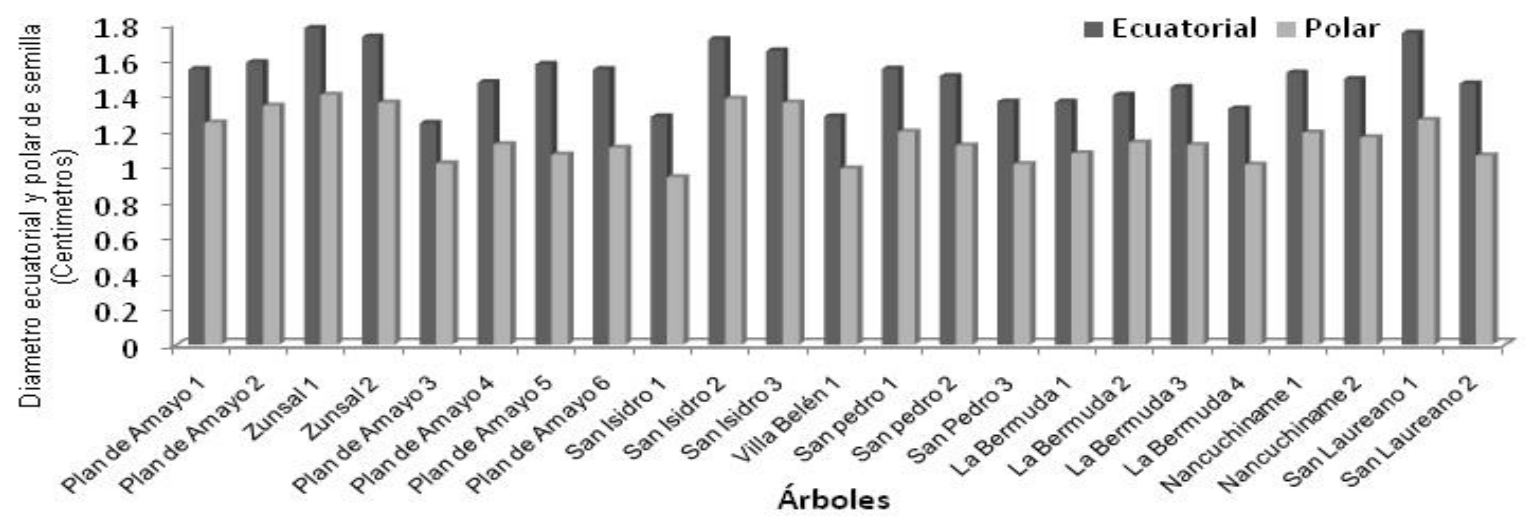

Figura 3. Diámetro ecuatorial y polar de fruto y semilla. Análisis descriptivo de variables cuantitativas (2013). 


\section{e. Peso de fruto y semilla}

El peso promedio de frutos de los 14 árboles fue de 3.58gr, con una desviación estándar de 0.46 gr y coeficiente de variación de $12.84 \%$. Por otra parte, el árbol Nancuchiname 1 es el que presentó el mayor peso, con 4.46gr, mientras que el menor valor lo mostró el árbol Plan de Amayo 3 con 2.71gr (Figura 4).

El 79\% de los árboles se encontraron dentro de un rango de 3.12 - 4.04gr. Una característica muy sobresaliente e importante del material Nancuchiname 1 es que presentó frutos con una y dos semillas. La presencia de esos frutos con dos semillas es la que influye directamente en el peso antes mencionado. Se tomaron frutos al azar - que reunieran esas características - para determinar el peso promedio. Dicha característica probablemente se debe a una mutación del árbol en su código genético.

En cuanto al peso de semilla, el árbol Plan de Amayo 2 presentó el valor más alto (1.94gr), mientras que el más bajo fue el árbol San Isidro 1 (1.11gr) (Figura 4). El peso promedio de los 23 árboles fue de 1.59gr, con una desviación estándar de 0.22 gr y un coeficiente de variación de $14 \%$. Por otra parte, el $70 \%$ de los árboles se encontró dentro del rango 1.37 - 1.81gr. Los resultados obtenidos son afines a los mencionados por CATIE (2003), en donde se menciona el valor de $1.11-0.83$ gr.

Gráfica sobre peso de semilla y fruto de árboles de Ojushte

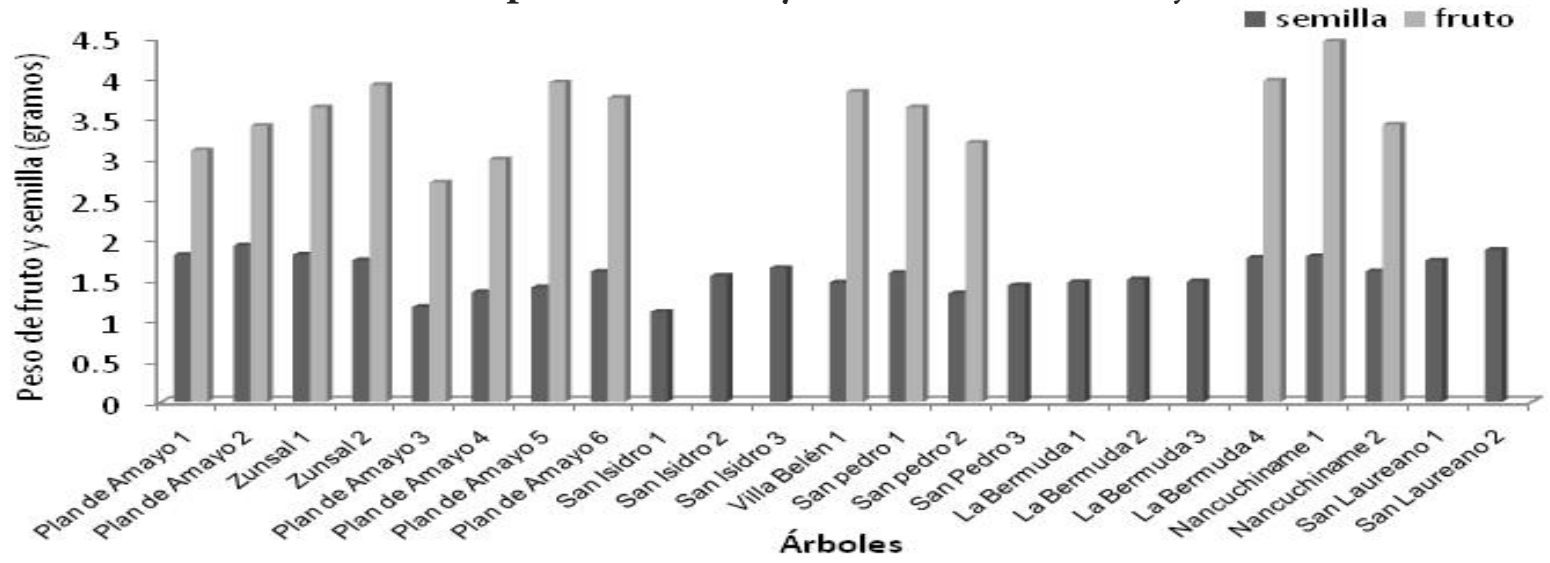

Figura 4. Detalle sobre datos de peso de semilla y fruto de Ojushte (2013).

\subsection{Análisis bromatológico}

\section{a. Porcentaje de proteína cruda}

El árbol San Isidro 3 presentó un 14.89\% en cuanto a proteína, mientras que el árbol Plan de Amayo 1 mostró un índice menor
(11.55\%) (Figura 5). Por otra parte, el contenido promedio de proteína en las semillas de los 23 árboles fue de $13.12 \%$, con una desviación estándar de $0.87 \%$; y coeficiente de variación de $6.63 \%$. Finalmente, el $65 \%$ de los árboles de Ojushte se encuentra dentro del rango 12.25 - $13.99 \%$. 
Similares valores menciona Asenjo et al, citado por Arévalo (2010), afirmando que la semilla de Ojushte se caracteriza por un alto contenido de proteína, el cual va desde un $10 \%$ hasta un $16 \%$. En comparación con otros granos, Bello, citado por Medina (2006), menciona que el grano de frijol - dependiendo de la variedad - puede presentar un valor de proteína entre el 16-30\%, mientras que el maíz posee un valor de $8.88 \%$, siendo un $4.24 \%$ más bajo que el promedio del Ojushte.

\section{b. Porcentaje de fibra cruda}

Al analizar la fibra cruda de las semilla, el árbol Plan de Amayo 6 presentó el valor más alto, con 14.20\%; por lo que el árbol Plan de Amayo 1 mostró un 7.05\% (Figura 5). El contenido promedio de fibra cruda en las semillas de los 23 árboles fue de 9.09\%, con una desviación estándar de $1.50 \%$ y coeficiente de variación de 16.50\%. En total, el $87 \%$ de los árboles se mantiene dentro del rango 7.59 - 10.59\%. Asenjo et al., citado por Arévalo (2010), menciona un rango más amplio al encontrado, pero que muy afín a las cifras. Este rango es de $4.6-20 \%$.

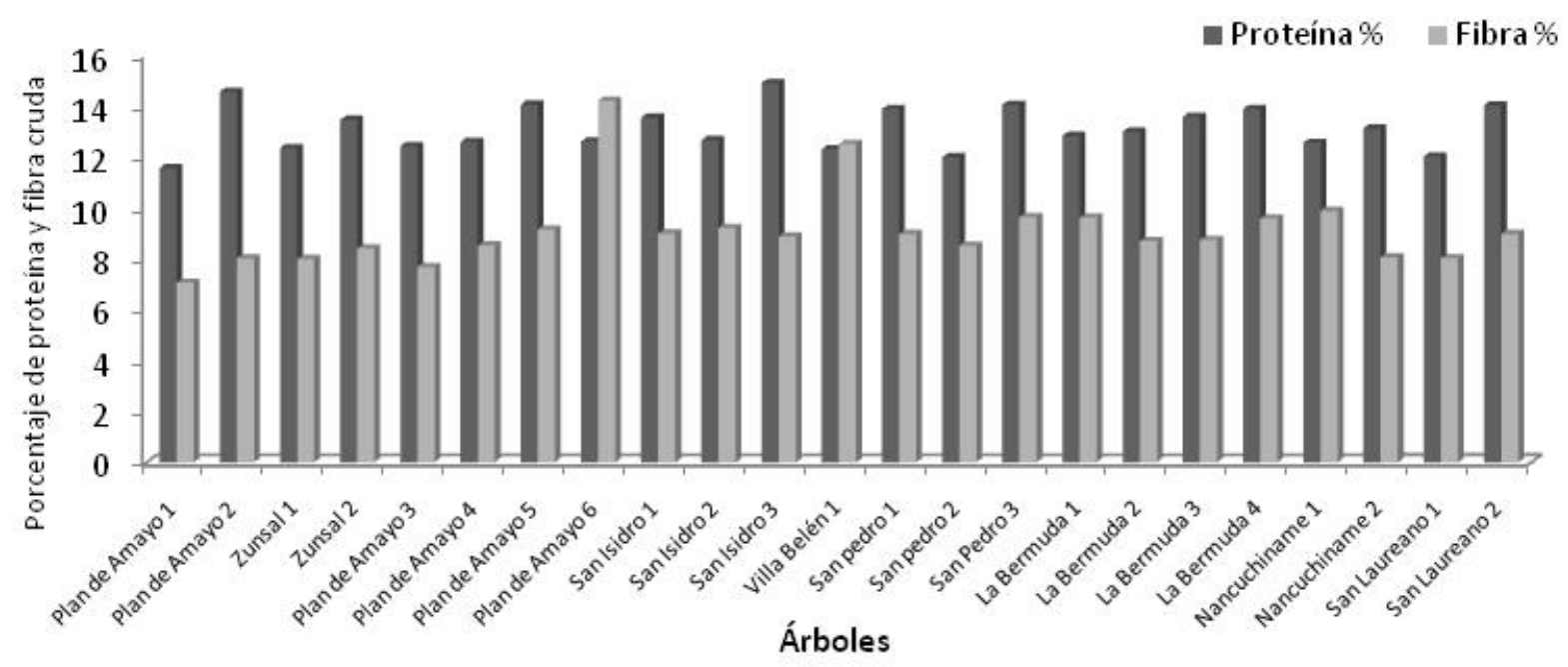

Figura 5. Niveles de proteína y fibra cruda en los 23 árboles de Ojushte estudiados. Análisis bromatológico (2013).

\section{c. Porcentaje de grasa}

El árbol Zunsal 1 presentó un 1.86\%, en comparación al árbol Nancuchiname 1 que tuvo un $0.24 \%$ (Figura 6). El contenido promedio de grasa en las semillas de los 23 árboles fue de $1.01 \%$, mientras que la desviación estándar fue de $0.33 \%$, con un coeficiente de variación de $32.67 \%$. En resumen, el 83\% de los 23 árboles estuvo dentro del rango $0.68-1.34 \%$. 
Gráfica sobre contenido de grasa en semilla de Ojushte

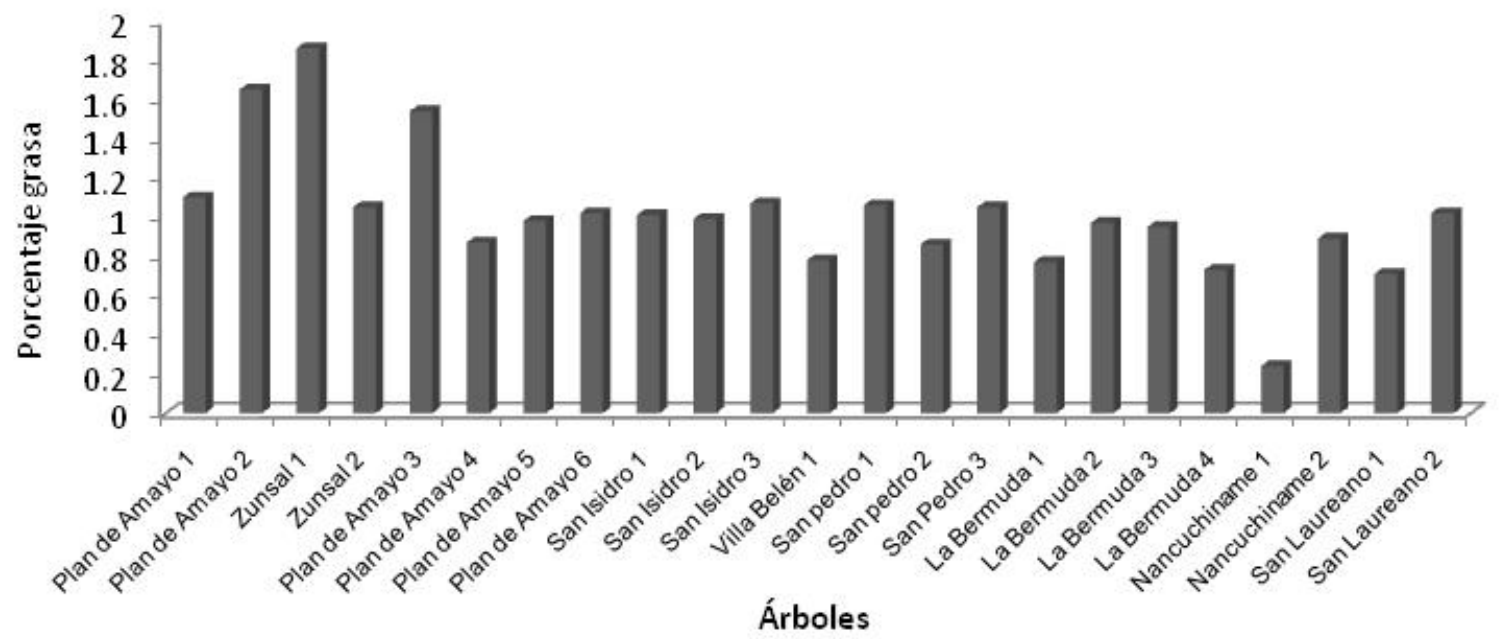

Figura 6. Niveles de contenido de grasa en semilla de árboles estudiados. Análisis bromatológico (2013).

\section{d. Contenido de hierro}

El árbol La Bermuda 1 presentó 111.13 mg.l-1, mientras que el valor más bajo correspondió al árbol San Isidro 3 con $28.47 \mathrm{mg} . \mathrm{l}^{-1}$ (Figura 7). El contenido promedio de hierro en las semillas de los 23 árboles fue de $52.48 \mathrm{mg} .1^{-1}$, con una desviación estándar de $21.61 \mathrm{mg} .1^{1-1}$, y un coeficiente de variación de $41.17 \%$. En ge- neral, el 70\% de los árboles se encontró dentro del rango $31-74.09 \mathrm{mg} . \mathrm{l}^{-1}$.

La importancia del hierro para el organismo humano es la participación en la producción de hemoglobina, la cual es el componente principal de los glóbulos rojos. Esta también contribuye en la formación de las proteínas musculares y a la metabolización de ciertas

\section{Gráfica sobre contenido de hierro en semilla de Ojushte}

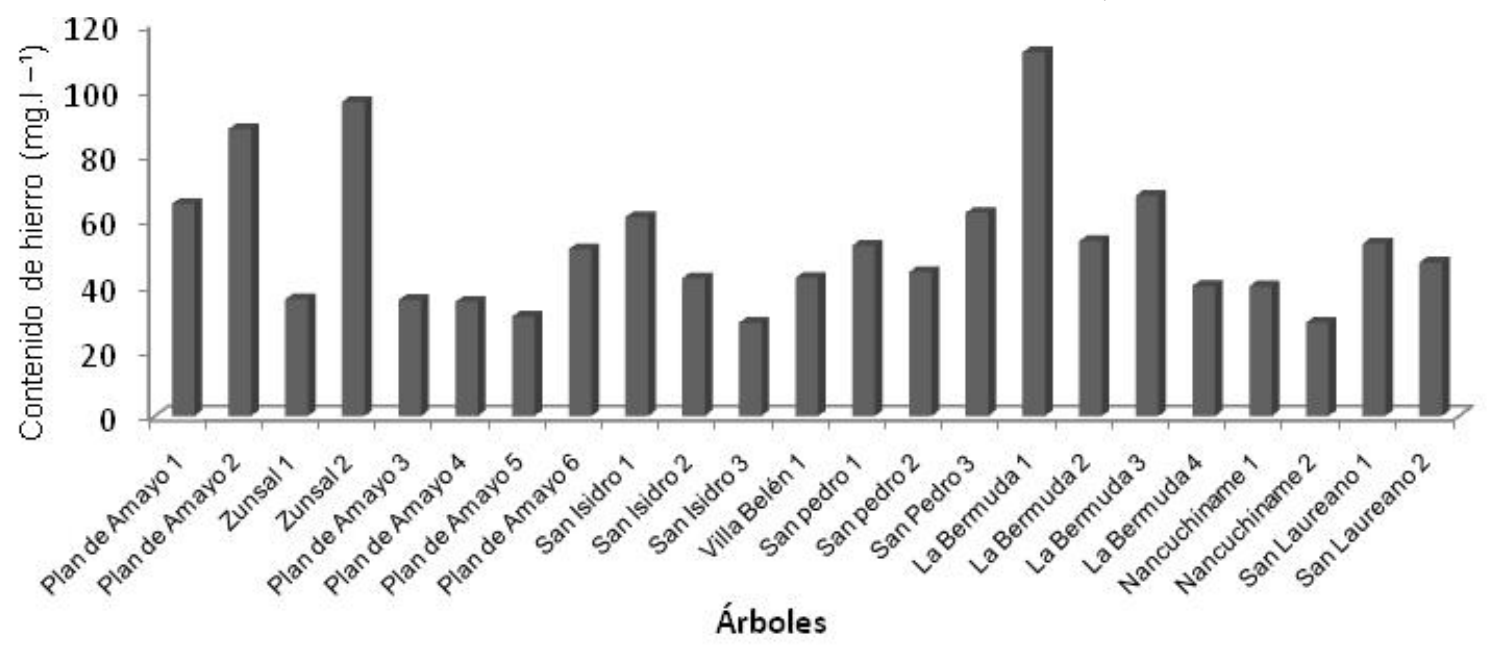

Figura 7. Niveles de hierro en los árboles estudiados. Análisis bromatológico (2013). 
enzimas del cuerpo. Asimismo, el hierro es un nutriente imprescindible para la liberación de oxígeno, brindando energía de las células del cuerpo y así eliminar el dióxido de carbono (Lelven 2012).

\section{e. Contenido de zinc}

El árbol Plan de Amayo 1 tuvo un contenido de zinc de $23.72 \mathrm{mg} . \mathrm{l}^{-1}$, siendo mucho más elevado que el nivel del árbol San Pedro 3

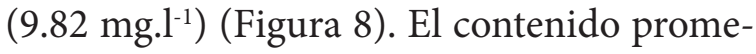

dio de zinc en las semillas de los árboles es-

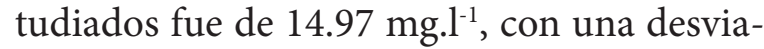
ción estándar de $3.37 \mathrm{mg} .1^{-1}$, y un coeficiente de variación de $22.51 \%$. En resumen, el 65\% de los árboles de Ojushte se encontró dentro del rango 11.6 - $18.34 \mathrm{mg} . \mathrm{l}^{-1}$.

El zinc es un mineral esencial para el cuerpo humano, ya que juega un papel clave en la división celular. Por esta razón, su presencia en cada célula del cuerpo es indispensable; asimismo, forma parte del sistema inmune, ayudando a evitar enfermedades (Valadés 2010).

\section{Gráfica que muestra el contenido de zinc en semilla del Ojushte}

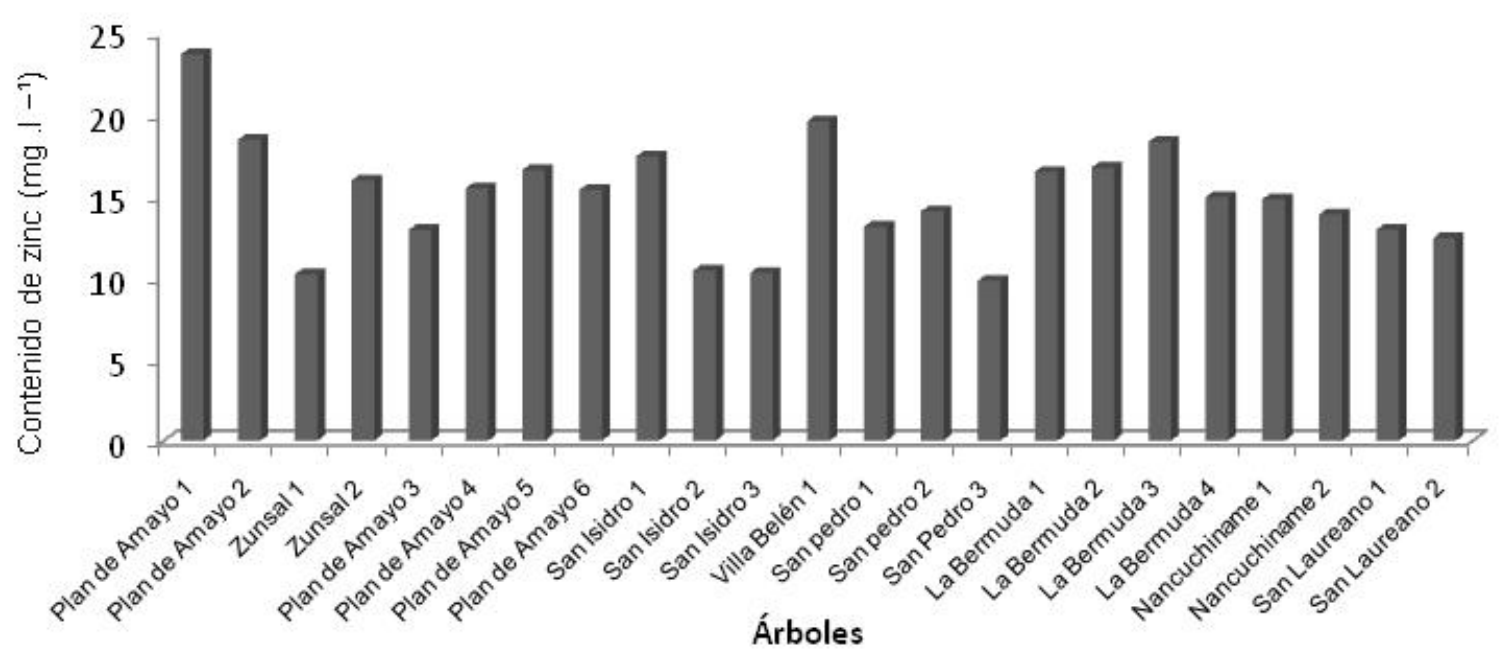

Figura 8. Niveles de zinc en las semillas de los árboles estudiados. Análisis bromatológico (2013).

\subsection{Análisis de correlación}

El largo de hoja presentó una correlación positiva con ancho de hoja $(r=0.74)$, con área foliar $(r=0.71)$. Esta última con ancho de hoja $(r=0.70)$. Esto indica que existe una simetría entre las variables; es decir, el aumento de una característica de la hoja durante el desarrollo, tiene un efecto directo y positivo sobre las otras dimensiones.

La correlación positiva de peso de semilla con diámetro ecuatorial $(r=0.60)$, a su vez con diámetro polar $(r=0.60)$, indica que el peso aumentó a medida que las dimensiones de la semilla se aumenta. Por otra parte, se observa 
una asociación positiva muy importante, la cual es el diámetro ecuatorial con diámetro polar, que presentó una correlación positiva alta $(r=0.89)$. Esto indica que, al aumentarse una dimensión del diámetro, la otra aumenta en la misma dirección, siendo altamente significativo (1\%).

En cuanto a componentes químicos, existe una correlación negativa entre ceniza y grasa, con un valor $(r=-0.53)$. Este valor indica que si uno de los componentes aumenta o se encuentra en mayor cantidad dentro de una semilla, el otro tiende a ser menor o a disminuir, siendo significativo al 5\%.

\subsection{Análisis de conglomerados}

Al ejecutar el análisis multivariado en los 23 árboles de Ojushte caracterizados, se encontraron seis grupos. Dentro de los grupos se evidenció homogeneidad y similitud; pero a su vez, existió heterogeneidad y diferencias entre los diferentes grupos.

El grupo uno, estuvo formado por los árboles: Plan de Amayo 4, Plan de Amayo 5, San Pedro 1, Plan de Amayo 1 y San Isidro 1 (Figura 9). Estos materiales fueron influenciados por las variables edad, DAP (diámetro a la altura del pecho), altura de árbol y diámetro de copa, debido a la similitud y homogeneidad existente entre las variables (estructura, simetría y arquitectura del árbol). Al mismo tiempo, este grupo sobresalió por mostrar el ma- yor contenido de zinc en semilla, las menores dimensiones de hoja (longitud, ancho y área), y el menor peso de semilla. En cuanto al resto de variables o descriptores, las accesiones tuvieron valores intermedios, a pesar de que el coeficiente de variación promedio para el grupo fue $12.16 \%$.

En el segundo grupo se observó la formación de dos subgrupos: el primer subgrupo conformado por los árboles Plan de Amayo $2 \mathrm{y}$ Zunsal 2; y el segundo subgrupo que correspondió al árbol La Bermuda 1 (Figura 9). Estos materiales estuvieron determinados por las variables: diámetro polar de semilla, diámetro ecuatorial y peso promedio. Probablemente su agrupación obedezca a similitudes existentes en las variables mencionadas.

Por otra parte, este grupo se caracterizó por presentar los valores más altos respecto a variables relacionadas a semilla (diámetro polar, diámetro ecuatorial, peso, contenido de proteína y contenido de hierro). Asimismo, el coeficiente de variación promedio para este grupo fue de $13.47 \%$.

En el tercer grupo también se formaron dos subgrupos: en el primero encontramos los árboles Plan de Amayo 3 y San Pedro 3; de igual forma encontramos en el segundo subgrupo el árbol Zunsal 1 (Figura 9). Estos fueron influenciados por las variables de largo de hoja, ancho de hoja y área foliar (su agrupación se debe a la similitud existente a dimen- 
siones de la hoja). Este grupo se caracterizó por presentar el mayor contenido de grasa, y el menor contenido de fibra, ceniza y zinc. Además, este grupo presentó un coeficiente de variación promedio de $14.82 \%$.

El cuarto grupo está formado únicamente por dos árboles: Plan de Amayo 6 y La Bermuda 4 (Figura 9). Estos árboles muestran similitud debido a las siguientes variables: hierro, ceniza, zinc y peso promedio. Este grupo se caracterizó por presentar los valores más altos en cuanto a edad, DAP (diámetro a la altura del pecho), altura, diámetro de copa, dimensiones de hoja (largo, ancho y área) y contenido de fibra en semilla. Mientras, que los valores más bajos se dieron respecto a diámetro polar y ecuatorial de semilla. El coeficiente de variación promedio para este grupo fue de $12.53 \%$.

El grupo cinco estuvo formado por los subgrupos de los árboles La Bermuda 2 y La Bermuda 3; y el grupo de los árboles San Isidro 2, Villa Belén 1 y San Isidro 3 (Figura 9). Para la formación de este grupo influyeron las variables humedad parcial, fibra y grasa. Este grupo se caracterizó por presentar los mayores valores respecto a humedad parcial y humedad total. El resto de las variables muestran valores intermedios, mientras que el coeficiente de variación para el grupo fue de $14.92 \%$.

El sexto grupo conformado por los dos subgrupos: el primero de los árboles Nancuchiname 1, Nancuchiname 2, San Laureano 2 y San Pedro 2; y el grupo del árbol San Laureano 1 (Figura 9). Este último grupo muestra similitud por la variable proteína. Por otra parte, se caracterizó por mostrar los valores más bajos en las variables: DAP (diámetro a la altura del pecho), altura, diámetro de copa, contenido de proteína en semilla, grasa y hierro; el coeficiente de variación para este grupo fue de $18.42 \%$. 


\section{Dendrograma que utiliza una vinculación de Ward: Combinación de conglomerados de distancias re-escalados}

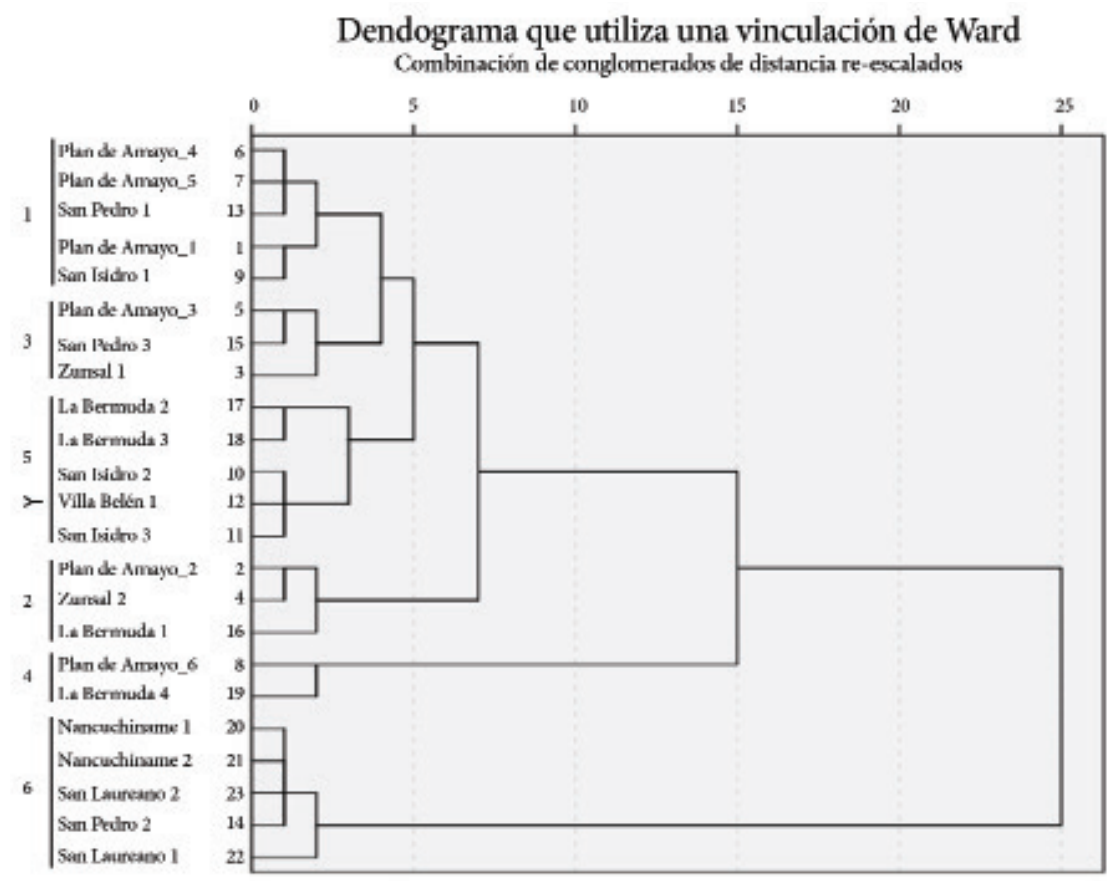

Figura 9. Dendrograma de distancias entre 23 materiales de germoplasma (2013).

\section{Conclusiones}

En el estudio se caracterizaron un total de 30 árboles productivos de Ojushte, de los cuales solamente 23 de ellos se encontraron con semilla, siendo estos a los que se le realizó análisis bromatológicos.

Es recomendable hacer una búsqueda más amplia de árboles de Ojushte a nivel nacional para caracterizarlos. A su vez, se propone crear jardines clónales de esta especie en peligro de extinción; ya que su presencia se reduce únicamente a parques cafetaleros, bosques de galería y áreas naturales protegidas.

El Ojushte se puede reproducir por medio de técnicas de injertación, específicamente aquellos árboles que presentaron características sobresalientes en cuanto a proteína, grasa, hierro y zinc en semilla. Posteriormente, los beneficios de este árbol se pueden promocionar en aquellas instituciones que fomentan programas de seguridad alimentaria en comunidades y/o recomendarlo dentro de sistemas de alta productividad.

- Como resultado de la caracterización morfoagronómica de las características cualitativas del Ojushte, se presentaron los siguientes resultados:

- El 63.30\% de los árboles presentaron forma de gamba IV, mientras que $3.30 \%$ presentaron una forma $\mathrm{I} y \mathrm{~V}$, respectivamen- 
te. Un $76.66 \%$ de los árboles presentaron un color de tronco café oscuro - grisáceo, y solamente el $23.34 \%$ color café claro - grisáceo.

- El periodo de fructificación para la especie es aproximadamente de 60 a 75 días. Por otra parte, el $73.4 \%$ de los árboles presentaron frutos verdes, el $13.30 \%$ frutos amarillos y un $13.30 \%$ frutos rojos. La producción de fruto y semilla de Ojushte ocurre de enero a agosto, pero los meses de mayor abundancia son de junio a julio. El 76.67\% de los árboles presentaron un envés de hoja escasamente pubescente, mientras que el $23.30 \%$ mostró un envés glabro.

- Como resultado de la caracterización morfoagronómica de caracteres cuantitativos (fruto y semilla), se determinaron parámetros o rangos (aplicando probabilidad estadística), que indican que al encontrar frutos y semillas de un árbol nuevo. La probabilidad de que el Ojushte se encuentre entre dichos rangos es del 65\% o más aproximadamente.

\section{Los rangos son:}

\section{a. Para características físicas de fruto} y semilla:

Diámetro de fruto: $1.69-2.28 \mathrm{~cm}$; peso de fruto: 3.12 - 4.04g; diámetro de semilla: 1.02 $-1.65 \mathrm{~cm}$ y peso de semilla: $1.37-1.81 \mathrm{~g}$.

\section{b. Para características químicas de semilla:}

Contenido de proteína: 12.25 - 13.99\%; contenido de fibra: 7.56 - 10.59\%; contenido de grasa: $0.68-1.34 \%$; contenido de zinc: 11.6 - $18.34 \mathrm{mg} . \mathrm{l}^{-1} \mathrm{y}$ contenido de hierro: 31 - 74.09 mg. ${ }^{-1}$

- La variabilidad genética cuantitativa de los árboles muestreados fue determinada por el $42 \%$ de los descriptores, mostrándose un coeficiente de variación mayor del 23\%.

- El método multivariado permitió agrupar en seis componentes a los árboles de Ojushte identificados. Esto propició que se tipificaran grupos o poblaciones específicas de Ojushte, ya que cada una de ellas se identifica mostrando caracteres muy afines entre sí y diferentes entre otros grupos.

Esto ratifica que el consumo de Ojushte es muy nutritivo, ya que presenta valores mayores a los del maíz y sorgo; ayudando incluso a mitigar problemas de desnutrición. 


\section{Referencias}

Aragón B., UR. (1990). Caracterización preliminar del ramón (Brosimum alicastrum swart), in situ en el bosque muy húmedo sub - tropical cálido de Peten Guatemala. Tesis Ing. Agr. Guatemala, USAC. $140 \mathrm{p}$

Arévalo S., AI. (2010). Respuesta Glicémica de la semilla del Ramón, Brosimum alicastrum en Mujeres de 16 a 25 años de edad, residentes de la Ciudad Capital, Guatemala. Tesis Lic. Guatemala, Universidad Rafael Landívar. p18

CATIE (Centro Agronómico Tropical de Investigación y Enseñanza) (2003). Árboles de Centroamérica: un manual para extensionistas. Eds. J. Cordero; DH. Boshier. Turrialba. CR. P 403 - 406

Lelven, R. (2012). Beneficios del hierro en el funcionamiento del cuerpo humano. Recuperado de http://otramedicina.imujer.com/4175/beneficios-del-hierro-para-la-salud

Linares L., C. E. e Ibáñez R., J. M. (1992). Influencia de la estratificación en la germinación y del ácido indolbutirico (AIB) en el enraizamiento del ojushte. Tesis lic. en biología. San Salvador, SV, UES. p 4

Medina H., M. D. (2006). Desarrollo de una barra nutricional a base de gránola y frijol rojo (Phaseolus vulgaris). Honduras, s.e. p 3

Meiners, M. y otros (2009). El ramón: fruto de nuestra cultura y raíz para la conservación. México, CONABIO. 10p

Mendoza G., M. y Santillana C., M. E. (2012). Reintroducción de Brosimum alicastrum (Moraceae) en el Ejido de Zenzontla en La Reserva de la Biosfera de la Sierra de Manantlán, México. Madrid, ES, s.e. p 4

Morales O., E. R; Herrera T., L. G. (2009). Ramón (Brosimum alicastrum Swartz.): Protocolo para su colecta, beneficio y almacenaje. Yucatán, MX, s.e. 18p

Valadés, J. (2010). Beneficios del zinc en el cuerpo humano (en línea). Recuperado de: http:// remediosnaturales.about.com/od/Investigacion-Cient ifica/t p/Be nef cios-Zinc.htm

Witsberguer; D. y otros (1982). Árboles del parquet Deininger. San Salvador, SV, Dirección de Publicaciones. $337 \mathrm{p}$ 